\title{
A TSSA Algorithm based Approach to Enhance the Performance of Warehouse System
}

\author{
F. T. S. Chan \\ Department of Industrial and Manufacturing Systems \\ Engineering, The University of Hong Kong \\ Pok Fu Lam Road, Hong Kong \\ e-mail: ftschan@hkucc.hku.hk
}

\author{
V. Kumar \\ Department of Management, \\ Exeter Business School, University of Exeter, \\ Exeter, EX4 4PU, United Kingdom \\ e-mail: vk211@exeter.ac.uk
}

\begin{abstract}
In this plethora of increased competitiveness and globalization the effective management of the warehouse system is a challenging task. Realizing that proper scheduling of the warehouses is necessary to outperform the competitors on cost, lead time, and customer service basis (Koster, 1998); the proposed research focuses on optimization of warehouse scheduling problems. This research aims to minimize the total tardiness so that the overall time involved in managing the inventory inside the warehouse could be effectively reduced. This research also deals with the vehicle routing issues in the warehousing scenario and considers various constraints, and decision variables, directly influencing the undertaken objective so as to make the model more realistic to the real world environment. The authors have also proposed a hybrid Tabu Sample-sort Simulated Annealing (TSSA) algorithm to reduce the tardiness as well as to enhance the performance of the warehousing system. The proposed TSSA algorithm inherits the merits of the Tabu Search and Sample-sort Annealing algorithm. The comparative analysis of the results of the TSSA algorithm with other algorithms such as Simulated Annealing (SA), Tabu Search (TS), and Hybrid Tabu Search algorithms indicates it's superiority over others, both in terms of computational time as well as total tardiness reduction.
\end{abstract}

Keywords - Warehousing, TSSA, Tardiness, Sample sort, Scheduling

\section{INTRODUCTION}

Warehousing issues has been of prime importance due to the reliability of the firm's supply chain on their overall efficacy. In the past decade manufacturing entities were more concerned about reducing the production lead time, flow time etc. However, in recent years the grown interest of the researchers in warehousing issues has generated curiosity world wide (Rubrico et al. 2006, Petinis et al. 2005, Lee \& Elsayed 2005, Duc \& Koester 2005, Heragu et al. 2003). Van der berg and Zijm (1999) explained the various types of the warehousing systems, and discussed the various decision problems encountered in setting up warehousing systems, including justification, design, planning and control issues. The warehouse location problems under continuous economies of scale were studied by Feldman et al. (1966). Warehousing efficiency is a key factor in the supply chain management to outperform the competitors on the basis of customer service, lead-times, and costs (Koster, 1998). The warehouse keeps the stocks of these products after production stage, and holds them till they are delivered to the market place. The delay in the delivery of the product may lead to the failure of the supply chains, as these chains are aimed for the proper and timely flow of the inventory. By efficient management of the inventory, both at the arrival end and the delivery end, the warehouse contributes towards the timely delivery of the product as per their demand in the market. The modern warehouses are equipped with the automated guided vehicles (AGVs) for picking up the products from the arrival end and to store them at specific locations, and then finally pick them up to the delivery end, when the demand for the specific products arrives. Even though the AGVs enhance the performance of the warehouses, the warehouse scheduling is a challenging and vital task. The allocation of the products to the specific places inside the warehouses, to assign an AGV for carrying a particular product, and priority to deliver the exact products are few important assignments that needs to be taken care off during the warehouse scheduling. A model for the warehouse picking was proposed by Daniels et al. (1998). They formulated a model for simultaneously determining the assignment and sequencing decisions, and compared it to previous models for order picking. The vehicle routing problem inside the warehouses has been studied by few researchers. The warehouse location-routing problem (WLRP) was studied by Perl and Daskin (1985). Burkard et al. (1995) analyzed, and optimized the vehicle routing in an automated warehouse. Molnar et al. (2002) presented a two-phase model for planning and scheduling of order picking processes. Route Generation for Warehouse Management using Fast Heuristics method was studied by Rubrico et al. (2004). They solved a sub-problem of warehouse scheduling involving the routing of intelligent agents as a preliminary step in optimizing the total schedule.

However, there have been few studies which integrate warehouse space allocation as well as AGV routing issues (Burkard et al., 1995, Ito and Abadi, 2002 etc.). To fill this research gap present study integrates and studies the warehouse scheduling along with the AGV allocation based case study in a randomly generated problem set environment. The present paper highlights the benefits associated with the efficient warehouse management, and proposes a hybrid Tabu Samplesort Simulated Annealing (TSSA) algorithm to solve the warehouse scheduling problem. The prime objective of the present research is the overall minimization of the tardiness encountered during the product transferring from the arrival to 
the delivery end. The idea behind proposing the new TSSA algorithm was to overcome the demerits associated with the conventional algorithms such as SA, and TS as well as to find an alternative algorithm that would be more efficient in solving the complex warehouse scheduling problems. The efficacy of the TSSA algorithm has been shown by comparing it against existing algorithms such as TS, SA, and HTSA.

The paper is organized as follows. Section II discusses the problem scenario, objective functions, constrains, as well as decision variables. Section III explains in detailed about the proposed TSSA algorithm i.e. the background of the algorithm and mentions the steps involved. Computational results have been discussed in section IV. And section V concludes the present work suggesting some future research directions.

\section{PROBLEM FORMULATION}

The present work considers a warehouse management model resembling with the existing warehousing scenarios in the industries. In the proposed warehouse model the batches of products are required to be efficiently stored, and dispatched as per their due dates. The model consists of various storage locations inside the warehouse. The proposed work also considers the Automatic Guided Vehicles (AGVs) that are used to pickup/load the inventory from the supplier end, store it to the desired location, and finally deliver it as per the their due dates. The batches are required to be stored into specific location inside the warehouse, and dispatched according to their assigned due dates. In the model the inventory batches are of different sizes, and each batch has its own due date. However, to reduce the complexity of the problem in the proposed work the shape and size of the product has been neglected, and uniform dimension of the products were assumed. The warehouse scheduling problem is aimed at minimization of the overall tardiness incurred to deliver the product. The proposed model imposes several constraints during the problem formulation to make the proposed work more realistic to the real environment. The products of each batch have some precedence relationship, and they are supposed to be handled accordingly.

The loading and unloading time has been uniformly distributed in the range of 4-8. The proposed work considers the handling of total 15 order batches. The total number of products that need to be handled is assumed to be 40 . The batches can be allocated inside the warehouse at 12 different locations. The distance between locations is uniformly distributed in the range of $30-60 \mathrm{~m}$. The total number of AGVs employed in the present problem is 9, that transfers these batches from the arrival end to storage locations, and finally to the delivery end. The speed of the AGV is kept constant at 50 $\mathrm{m} / \mathrm{min}$. The mathematical modeling has been explained in the subsections discussed below.

\section{A. Parameters}

$\begin{array}{ll}\mathrm{t} & =\text { time period } \\ \mathrm{j} & =\text { job } \\ \mathrm{u}, \mathrm{v} & =\text { locations } \\ \mathrm{r} & =\text { vehicle }\end{array}$

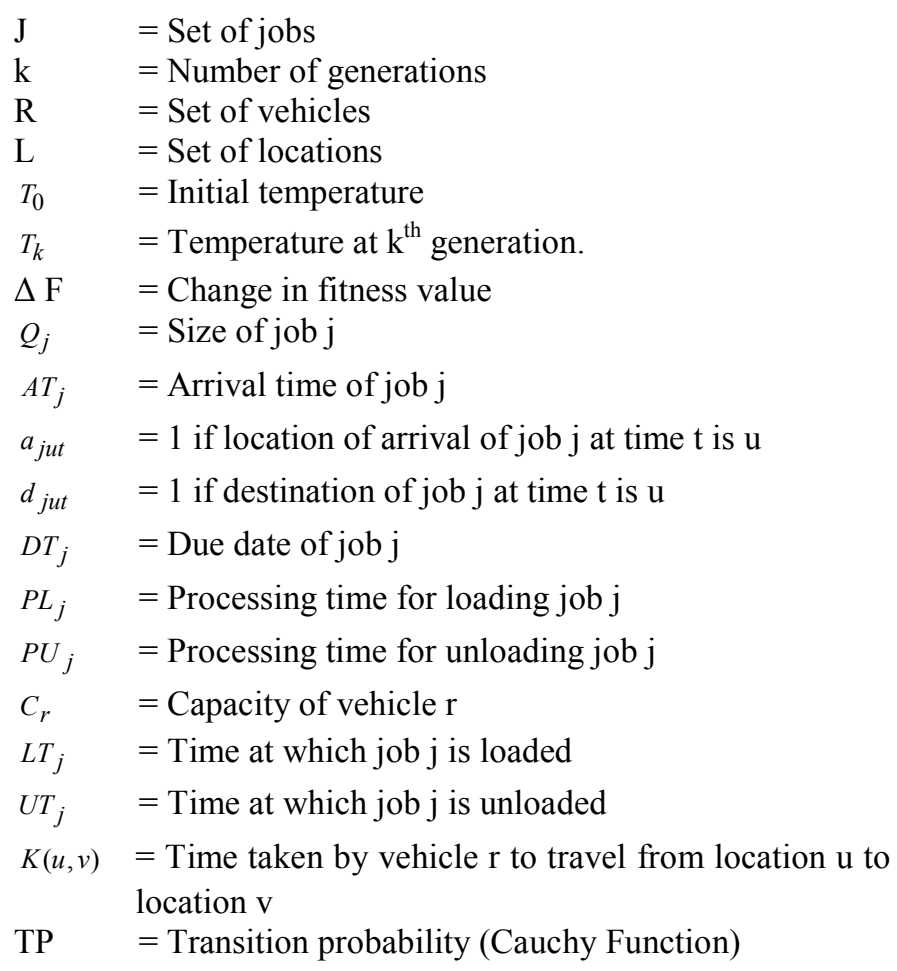

\section{B. Decision Variables}

$Y_{j r}= \begin{cases}1 & \text { if } \text { job } j \text { is loaded on vehicle } r \\ 0 & \text { otherwise }\end{cases}$
$\left[S_{\text {rut }}=\left\{\begin{array}{ll}1 & \text { if location of vehicle } r \text { at time } t \text { is } u \\ 0 & \text { otherwise }\end{array}\right]\right.$

\section{Objective Function}

The present research aims towards the overall reduction of the tardiness considering the due dates met during the effective warehouse management. The reduced tardiness leads to the timely delivery of the products, thus avoiding loss of the profit. The tardiness minimization equation can be represented as:

$\left[\operatorname{Min} \sum_{j \in J} \sum_{r \in R} Y_{j r} \max \left(U T_{j}-D T_{j}, 0\right)\right]$

The constraints considered in the proposed problem have been discussed below.

\section{Constraints}

(i) Vehicle capacity during loading

$\left[\sum_{t \in T} \sum_{u \in L} \sum_{j \in J} a_{j u t} Y_{j r} Q_{j} \leq C_{r} \quad \forall r \in R\right]$

(ii) Vehicle capacity during unloading

$\left[\sum_{t \in T} \sum_{u \in L} \sum_{j \in J} d_{j u t} Y_{j r} Q_{j} \leq C_{r} \quad \forall r \in R\right]$ 
$L T_{j} \geq A T_{j} \quad \forall j \in J$

(iv) Precedence of loading and unloading

$L T_{j}+P L_{j}+\sum_{r \in R} Y_{j r} k_{r}\left(a_{j}, d_{j}\right)+P U_{j} \leq U T_{j} \quad \forall j \in J$

(v) One vehicle can only load one job at a time

$\left(L T_{j}-L T_{i}\right)\left(L T_{j}+P L_{j}-L T_{i}\right)>0 \quad \forall j, i \in J, i \neq j$

(vi) One vehicle can only unload one job at a time

$\left\lfloor\left(U T_{j}-U T_{i}\right)\left(U T_{j}-P L_{j}-U T_{i}\right)>0 \quad \forall j, i \in J, i \neq j\right\rfloor$

(vii) Vehicle $\mathrm{r}$ is at the same location where job $\mathrm{j}$ is loaded

$\sum_{r \in R} Y_{j r} S_{r u t} a_{j u t}=1 \quad \forall r \in R, L T_{j} \leq t \leq L T_{j}+P L_{j}$

(viii) Vehicle $\mathrm{r}$ is at the same location where job $\mathrm{j}$ is unloaded.

$\sum_{r \in R} Y_{j r} S_{r u t} d_{j u t}=1 \quad \forall r \in R, U T_{j}-P U_{j} \leq t \leq U T_{j}$

The constraints (4) and (5) represents that the AGVs can't exceed their capacity during the loading or unloading. Constraint (6) explains that the arrival time can't be larger than the leaving time for a particular job. Constraints for the precedence relation between loading and unloading are presented by equation (7). The capacity restrictions have been imposed by the constraint shown in equation (8) and (9). According to this the AGV can load/unload only one job at a time. The location of the vehicle during loading and unloading has been fixed according to the position of the job as represented by the constraint in equation (10) and (11). The TSSA algorithm has been discussed in the next section.

\section{TSSA ALGORITHM}

Tabu Sample-sort Simulated Annealing (TSSA) algorithm encapsulates the prominent features of the Tabu search (TS), as well as Sample - Sort Annealing (SSA) algorithm. The idea/motivation behind the development of the proposed TSSA algorithm was to overcome the drawbacks that were inherited in TS (Glover, 1990), and Sample-Sort Annealing (SSA) algorithm (Thompson et al., 2005). Despite of the merits associated with TS and SSA in exploring the search space and solving the number of combinatorial optimization problems, few drawbacks linked with them motivated us to develop another robust method. TS start with a single point hence, its convergence speed, and final solution depends on the original point. The cycling-loop also is often encountered if the search moves to a previously visited solution that has not been existed in Tabu list for the last two iterations. Even SSA is more efficient than the conventional Simulated Annealing (SA) (Kirkpatrick et al., 1983) algorithm but it does not have any memory list of recently visited solution hence, there is a possibility of visiting the previously visited solution. Therefore, additional numbers of iterations are required to converge towards optimality which increases the computational time. In the proposed TSSA algorithm in order to reduce the computational time a fuzzy logic controller (FLC) (Kim et al., 2003) has been introduced in TSSA for adaptive change in swapping rate.

Inspired by the fact that efficient management of the warehouse system reduces the cost and lead times, as well as enhances the customer services, TSSA algorithm has been applied to enhance its efficacy. The way in which the TSSA algorithm operates has been presented in steps as described in the following subsection.

\section{A. Steps of TSSA}

Step 1. Generate $\mathrm{m}(\mathrm{m}=10)$ initial samplers $\left(\mathrm{x}_{1}, \mathrm{x}_{2}, \mathrm{x}_{3} \ldots \mathrm{x}_{\mathrm{m}}\right)$. In the proposed algorithm random initialization technique has been used. These samplers are generated according to the following steps:

$>$ Randomly select one operation having no predecessors.

$>$ Among the remaining operations, randomly select the another operation which has no predecessors

$>$ Repeat the above step until all operation has been selected.

$>$ Revisit all selected operations started from first operation and randomly assigned work station and $\mathrm{AGV}$ from the existing alternative

$>$ Repeat the above step until an appropriate work station and $\mathrm{AGV}$ are assigns to each selected operations.

$>$ Repeat all the abovementioned steps until prescribed numbers of chromosomes are generated

Step 2. The individuals are evaluated and their fitness are measured

Step 3. Initialization: Iteration $\mathrm{i}=1$, Tabu list $(\mathrm{TL})=\Phi$, reject $=0$, best solution $\left(\mathrm{x}_{\mathrm{b}}\right)=\mathrm{x}$; for each sampler a static temperature or initial temperature is assigned. Let the static temperatures are $\mathrm{T}_{1}, \mathrm{~T}_{2}, \mathrm{~T}_{3} \ldots \mathrm{T}_{\mathrm{m}}$. For $1,2,3 \ldots \mathrm{m}$ samplers respectively. The calculation of static temperatures is delineated in the next step.

Step 4. Following Steps are followed to determine the static temperature for each sampler;

Step 4.1 For each sampler generate 100 random solutions.

Step 4.2 Evaluate objective function for all the solutions and estimate the average fitness value ' $\lfloor\bar{f}\rfloor$ '.

Step 4.3 Calculate the standard deviation ' $[\sigma]$ 'considering the fitness value of each operation.

Step 4.4 Using the value of standard deviation and average fitness value, energy is calculated. The following formula is applied for calculation of energy.

$$
\lfloor\Delta E=\bar{f}+\sigma\rfloor
$$

Step 4.5 For determining the highest temperature ' $\left[T_{H}\right]$ ', following formula is employed:

$$
p=\left[\exp \left(-\frac{\Delta E}{T_{H}}\right)\right]
$$

Where $[p=0.75],\left[T_{H}\right]=$ highest temperature

Step 4.6 Arrange the solution in the ascending order according to their fitness values. The energy $([\Delta E])$ is calculated by taking the difference between highest fitness value and fitness value of next solution in the order.

Step 4.7 For determining the lowest temperature $\left(\left[T_{L}\right]\right)$, the following expression is implemented:

$$
\left[p=\exp \left(-\frac{\Delta E}{T_{L}}\right)\right]
$$

Where $[p=0.01],\left[T_{L}\right]=$ Lowest Temperature 
Step 4.8 Static temperature of $\mathrm{k}^{\text {th }}$ sampler can be found out by the following formula:

$$
\left\lfloor T_{k}=T_{H} \alpha^{m-k}\right\rfloor
$$

And $[l \leq k \leq m]$. It is monotonically increasing function.

Where $\alpha=$ cooling co-efficient

Step 4.10 For estimating the value of cooling co-efficient ( $[\alpha]$ ), first fix the temperature $\left[T_{L}\right]$ and $\left[T_{H}\right]$ for first and last sampler and use the following expression:

$$
\left\lfloor T_{L}=T_{H} \alpha^{m-l}\right\rfloor
$$

Step 5. For updating the samplers or generation of the neighboring samplers, a perturbation scheme is employed.

Step 6. The feasibility of the new generated solution is also checked and if the generated solution is feasible than go to step 7 else go to step 5 for generation of a feasible solution.

Step 7. Check the solution by Tabu list. If the neighboring solution exists in Tabu list then go to step 8 otherwise go to step 9.

Step 8. Check the solution by aspiration value. If the fitness value of new generated solution is higher than aspiration value, go to next step otherwise go to step for the generation of a new solution.

Step 9. Calculate the difference $([\Delta E])$ between the previous and its neighboring solution's fitness values. Let the initial solution is ' $\mathrm{x}$ ' and the solution generated by perturbation is $\left[x^{\prime}\right]$. Energy $([\Delta E])$ can be calculated as follows:

$$
\left[\Delta E=f\left(x^{\prime}\right)-f(x)\right]
$$

If the value of $[\Delta E \geq 0]$, go to next step otherwise go to step 11 .

Step 10. Replace the previous solution ' $\mathrm{x}$ ' by its neighboring solution $\left[x^{\prime}\right]$.This solution is also included in the Tabu list. The aspiration value is updated by the fitness value of the solution s', a perturbated solution.

Step 11. If the difference $\left[\Delta E_{b}\right]$ between the fitness values of perturbated solution and the best solution is greater than 0 , then go to next step else go to step 16. it is mathematically shown as follows:

$$
\left[\Delta E_{b}=f\left(x^{\prime}\right)-f\left(x_{b}\right)\right]
$$

Where, $\left[x^{\prime}\right]=$ perturbated solution, $\left[x_{b}\right]=$ best solution

Step 12. Replace the best solution $\left[x_{b}\right]$ by the perturbated solution $\left[x^{\prime}\right]$ and go to step 16 and assign zero value to reject.

Step 13. Calculate the probability by using the following equation:

$$
\left[p=\exp \left(-\Delta E / T_{k}\right)\right]
$$

Where, $\mathrm{T}_{\mathrm{k}}$ is the temperature at $\mathrm{k}^{\text {th }}$ iteration. Now a random number $\left(R_{a}\right)$ is generated between $(0,1)$. If the calculated probability is less than random number, go to step 15 else go to next step.

Step 14. Replace the existing solution by the perturbated solution and it is included in the Tabu list and updates the aspiration value by replacing it with perturbated solution's fitness value.

Step 15. If the probability is less than random number, reject the solution and increase the number of reject by one. If reject reaches to a pre determined maximum limit (in this paper, it is taken as 4), then go to step 20 other wise go to step 16 .

Step 16. Now a Fuzzy Logic Controller (FLC) is used for regulation of the swapping rate. This regulation will be adaptive in nature. The regulation by FLC has been discussed in detailed in Chan et al. (2006).

Step 17. The samplers are swapped according to updated swapping rate.

Step 18. Increase the number of iterations by one and update the temperature according to equation 22. If the number of iterations is not equal the maximum number of iterations, repeat step 5 to step 17 otherwise go to next step.

Step 19. Stop the iteration for that sampler, if reject for all samplers has reached on its maximum limit, go to next step else go to step 18 .

Step 20. Stop the iteration and $\left[x_{b}\right]$ is the optimal or near optimal solution.

The proposed TSSA algorithm works on the basis of the

\begin{tabular}{|c|c|}
\hline Parameters & Values or Range \\
\hline Number of batches to be handled & 15 \\
\hline $\begin{array}{c}\text { Number of products to be } \\
\text { handled (j) }\end{array}$ & 40 \\
\hline Number of AGVs employed & 9 \\
\hline $\begin{array}{l}\text { Number of warehouse locations } \\
\qquad(\mathrm{u}, \mathrm{v})\end{array}$ & 12 \\
\hline $\begin{array}{c}\text { Processing tine for loading and } \\
\text { unloading }\end{array}$ & Uniform $(4,8)$ \\
\hline Speed of AGV $\left(\mathrm{S}_{\mathrm{agv}}\right)$ & $50 \mathrm{~m} / \mathrm{min}$ \\
\hline Distance Between locations & Uniform $(30,60) \mathrm{m}$ \\
\hline \multirow[t]{3}{*}{ Arrival time $\left(A T_{j}\right)$} & $\sum_{u=0}^{V} s_{u j}$ \\
\hline & $\frac{u-v}{S_{a g v}}$ \\
\hline & $\begin{array}{l}\text { distance traveled to reach the } \\
\text { location } \mathrm{u} \text { for job } \mathrm{j}\end{array}$ \\
\hline
\end{tabular}
abovementioned steps.

\section{COMPUTATIONAL RESEULTS}

The present work proposes a new hybrid TSSA algorithm

Table 1: Problem Generation Scheme

Table 2: Due dates corresponding to the orders and their precedence relationship

\begin{tabular}{cccc}
\hline $\begin{array}{c}\text { Order } \\
\text { (Batches) }\end{array}$ & $\begin{array}{c}\text { Number product to } \\
\text { be transfer }\end{array}$ & $\begin{array}{c}\text { Precedence relation } \\
\text { between products }\end{array}$ & $\begin{array}{c}\text { Due } \\
\text { date }\end{array}$ \\
\hline 1 & 3 & $1-2-3$ & 55 \\
2 & 3 & $4-5-6$ & 65 \\
3 & 2 & $7-8$ & 75 \\
4 & 3 & $9-10-11$ & 88 \\
5 & 3 & $12-13-14$ & 67 \\
6 & 3 & $15-16-17$ & 57 \\
7 & 3 & $18-19-20$ & 60 \\
8 & 1 & 21 & 58 \\
9 & 3 & $22-23-24$ & 79 \\
10 & 3 & $25-26-27$ & 87 \\
11 & 3 & $28-29-30$ & 76 \\
12 & 2 & $31-32$ & 67 \\
13 & 3 & $33-34-35$ & 78 \\
14 & 2 & $36-37$ & 80 \\
15 & 3 & $38-39-40$ & 65 \\
\hline
\end{tabular}


to resolve the computational complexity involved in warehouse scheduling problems. In order to test the robustness of the proposed algorithm it has been tested on a model closely resembling with real environment scenario formulated in previous section. The proposed TSSA algorithm has been compared with other existing algorithms such as TS, SA, and HTSA algorithms to show its efficacy.

Table 3: Alternative warehouse where we can put our product during transfer process

\begin{tabular}{|c|c|c|c|c|}
\hline Product & Alternative 1 & Alternative 2 & Alternative 3 & Alternative 4 \\
\hline 1 & 6 & 12 & 2 & 8 \\
\hline 2 & 7 & 2 & 6 & 5 \\
\hline 3 & 4 & 10 & 11 & 7 \\
\hline 4 & 3 & 4 & 4 & 2 \\
\hline 5 & 3 & 7 & 9 & 4 \\
\hline 6 & 1 & 12 & 7 & 10 \\
\hline 7 & 5 & 8 & 12 & 12 \\
\hline 8 & 7 & 3 & 1 & 9 \\
\hline 9 & 7 & 12 & 8 & 5 \\
\hline 10 & 2 & 1 & 3 & 7 \\
\hline 11 & 1 & 8 & 12 & 3 \\
\hline 12 & 6 & 2 & 1 & 4 \\
\hline 13 & 5 & 7 & 8 & 10 \\
\hline 14 & 9 & 4 & 7 & 8 \\
\hline 15 & 7 & 3 & 2 & 6 \\
\hline 16 & 12 & 3 & 1 & 2 \\
\hline 17 & 8 & 1 & 6 & 11 \\
\hline 18 & 3 & 5 & 5 & 4 \\
\hline 19 & 12 & 7 & 8 & 9 \\
\hline 20 & 1 & 7 & 2 & 6 \\
\hline 21 & 8 & 2 & 10 & 3 \\
\hline 22 & 12 & 1 & 6 & 5 \\
\hline 23 & 2 & 6 & 7 & 8 \\
\hline 24 & 10 & 5 & 4 & 2 \\
\hline 25 & 4 & 9 & 3 & 1 \\
\hline 26 & 3 & 6 & 10 & 9 \\
\hline 27 & 1 & 2 & 3 & 7 \\
\hline 28 & 8 & 2 & 7 & 12 \\
\hline 29 & 4 & 1 & 11 & 6 \\
\hline 30 & 12 & 11 & 4 & 8 \\
\hline 31 & 8 & 7 & 6 & 9 \\
\hline 32 & 12 & 3 & 4 & 6 \\
\hline 33 & 11 & 1 & 3 & 2 \\
\hline 34 & 4 & 8 & 1 & 10 \\
\hline 35 & 6 & 4 & 8 & 11 \\
\hline 36 & 2 & 12 & 4 & 9 \\
\hline 37 & 2 & 8 & 12 & 3 \\
\hline 38 & 1 & 12 & 8 & 5 \\
\hline 39 & 11 & 5 & 12 & 4 \\
\hline 40 & 7 & 4 & 5 & 1 \\
\hline
\end{tabular}

Due to the lack of research involving the warehousing issues the standard data for the observed variables were quite difficult to find, hence to overcome this, a problem generation scheme was formulated which has been presented in Table 1 . The due date corresponding to the orders is shown in Table 2, along with the number of products to be handled, and their corresponding precedence relationships. The alternative warehouse locations, where the products can be allocated while transferring from arrival end to the delivery end, is presented in Table 3. The optimal sequence, their corresponding warehouse

\begin{tabular}{|c|c|}
\hline Product order: & $\begin{array}{llllllllllllll}7 & 36 & 28 & 33 & 34 & 38 & 39 & 8 & 22 & 40 & 21 & 15 & 16 \\
35 & 18 & 19 & 20 & 12 & 13 & 25 & 26 & 27 & 9 & 10 & 11 & 17 \\
29 & 30 & 37 & 31 & 32 & 4 & 5 & 6 & 1 & 2 & 3 & 23 & 24 & 14\end{array}$ \\
\hline Corresponding warehouse & $\begin{array}{llllllllllllllllll}8 & 7 & 7 & 2 & 4 & 9 & 8 & 12 & 7 & 2 & 8 & 2 & 8 & 4 & 7 & 1 & 6 & 4 \\
9 & 1 & 10 & 6 & 7 & 2 & 9 & 3 & 1 & 2 & 11 & 12 & 6 & 12 & 2 & 4 \\
11 & 4 & 12 & 6 & 12 & 5 & & & & & & & & & \end{array}$ \\
\hline Corresponding $A G V$ & $\begin{array}{llllllllllllllllll}1 & 3 & 4 & 5 & 2 & 8 & 4 & 3 & 5 & 9 & 3 & 8 & 2 & 1 & 7 & 2 & 9 & 4 \\
6 & 8 & 1 & 3 & 5 & 1 & 4 & 7 & 1 & 6 & 7 & 9 & 6 & 2 & 4 & 5 & 7 & 8 \\
2 & 3 & 1 & & & & & & & & & & & & & & & \end{array}$ \\
\hline
\end{tabular}

allocations, and corresponding AGVs employed, as finally obtained by the proposed TSSA algorithm has been shown in Table 4. The TSSA algorithm was compared with other existing algorithms to check its efficacy and robustness and presented in Table 5.

Table 5: Comparative Result in terms of the total tardiness

\begin{tabular}{cccc}
\hline $\begin{array}{c}\text { Algorithm } \\
\text { applied }\end{array}$ & $\begin{array}{c}\text { Computational Time } \\
\text { (seconds) }\end{array}$ & $\begin{array}{c}\text { No. of } \\
\text { Iterations }\end{array}$ & $\begin{array}{c}\text { Total } \\
\text { Tardiness }\end{array}$ \\
\hline SA & 22 & 656 & 38 \\
TS & 19 & 528 & 33 \\
HTSA & 11 & 412 & 30 \\
TSSA & 9 & 310 & 26
\end{tabular}

The comparative result from the SA, TS, and HTSA shows that the proposed algorithm over performs in terms of reduction of the overall tardiness. The result shows that the tardiness comes out to be 26 in case of TSSA, whereas the worst result is predicted by the SA algorithm i.e. 38. The TSSA algorithm

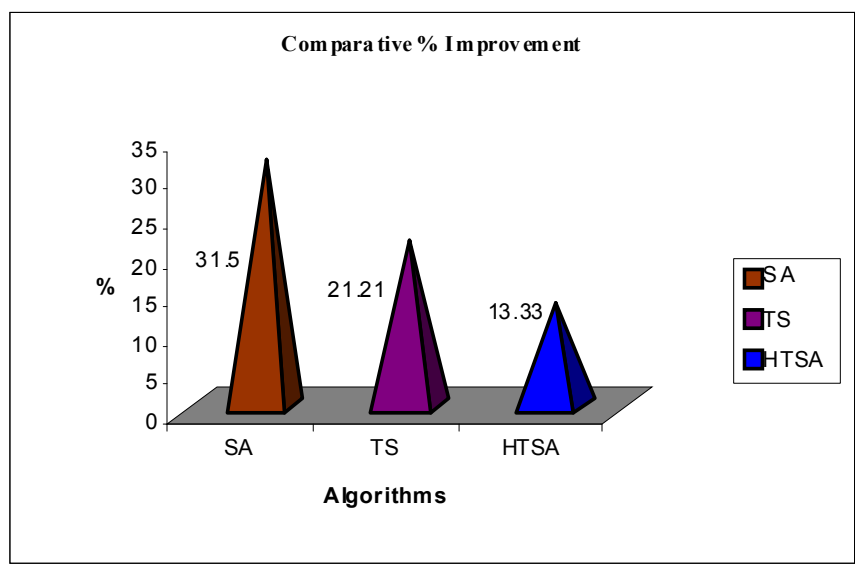

Figure 1. Comparitive percentage improvement wih other algorithms performs better than the most of the other methods in terms of both, the number of iteration required to reach the optimal 
solution, and the total computational time. The comparative percentage improvements obtained by TSSA algorithm with the other scheduling methods are shown in Figure (1). The comparative plot shows that the result from TSSA algorithm is $31.5 \%$ better than SA, $21.21 \%$ better than TS, and $13.33 \%$ better than HTSA algorithm. These results show that the proposed TSSA algorithm is more efficient in solving the complexity of the warehouse scheduling problem, compared to other methods. These finding suggests that the proposed TSSA algorithm performs much better than the other existing methods (SA, TS, and HTSA). The encouraging results demand its applicability in solving other complex scheduling problems. The TSSA algorithm needs to be tested on other various problems under different scenarios to validate its applicability in diverse field of manufacturing environment. Further research is required to enhance its robustness so that it could be efficiently applied to solve diverse type of problems. The applicability of TSAA algorithm apart from solving the scheduling problems will enhance its broad domain of tackling diverse problems. The next section concludes the present work and suggests some future work which researchers should focus in order to widen the applicability of TSSA under different circumstances.

\section{CONCLUSIONS}

The demand imposed by the present plethora to optimize the efficiency of the warehouse system has accelerated our research to focus on this critical issue. The present work attempts to optimize the efficacy of the warehousing system by its efficient scheduling and management. A hybrid TSSA algorithm has been proposed to solve the complex task of scheduling under warehousing scenario. The paper constructs a model closely resembling to the real warehousing scenario and discusses the various constraints, and decision variables, which have direct influence over the undertaken objective of reducing tardiness. The primary objective of this research was the overall reduction of the tardiness, in order to avoid any delay in the product delivery. The comparative analysis of the TSSA algorithm with TS, SA, and HTSA algorithms shows its supremacy over the others both is terms of tardiness reduction as well as computational time. Therefore, the reduction of tardiness leads to overall efficient supply chain management as well as it contributes to profitability and builds customer loyalty.

Though the present research attempts to discuss some critical issues related to warehouse management, more research work needs to be carried out to deal this issue in more detailed manner. Warehouse management is an important and hot issue to be discussed by the researchers these days hence; realizing its importance in supply chain and lack of significant research in this area, warehouse management leaves the possibility of plenty of quality research work for the researchers in near future. The future research may involve consideration of dimensions of the job during problem formulation to make it more realistic which was neglected in this present work. The work also needs to be aimed towards enhancing the robustness and accuracy of the algorithm. Although, Fuzzy Logic Controller (FLC) was included in present work to reduce the computational time significantly; forthcoming era will demand further reduction of computational time. The TSSA algorithm also needs to be tested under diverse and more computationally complex problems scenario to explore its applicability and efficacy.

\section{REFERENCES}

[1] R. E. Burkard, B. Fruhwirthy, and G. Rote,"Vehicle Routing in an Automated Warehouse: Analysis and Optimization, Appeared in Annals of Operations Research, 57, 1995, pp. 29-44

[2] F.T.S. Chan, V. Kumar, M. K. Tiwari, The relevance of outsourcing and leagile strategies in performance optimization of an integrated process planning and scheduling model, International Journal of Production Research, In press

[3] R. L. Daniels, J. L. Rummel, and R. Schantz, "A model for warehouse order picking", European Journal of Operational Research, 105, 1998, pp.1-17

[4] T. L. Duc, and R. B. M. (M.) De Koster, "Travel distance estimation and storage zone optimization in a 2-block class-based storage strategy warehouse", International Journal of Production Research, 43 (17), 2005 , pp. $3561-3581$

[5] E. Feldman, F. A. Lehrer, and T. L. Ray, "Warehouse location under continuous economies of scale", Management Science, 12(9), 1966, pp. 670-684

[6] F. Glover, "Artificial Intelligence, Heuristic Frameworks and Tabu Search", Managerial and Decision Economics, 11 (4), 1990, pp. 365-375

[7] S.S. Heragu, B. Kim, R.J. Graves, A. St Onge, "A hybrid scheduling and control system architecture for warehouse management", IEEE Transactions on Robotics and Automation, 16(6), 2003, pp. 991-1001

[8] T. Ito, and S. M. M. J. Abadi, "Agent-based material handling and inventory planning in warehouse", Journal of Intelligent Manufacturing, 13 (3), 2002, pp. 201-210.

[9] K. W. Kim, M. Gen, and G. Yamasaki, "Hybrid genetic algorithm with fuzzy logic for resource constrained project scheduling", Applied Soft Computing, 2/3 F, 2003, pp. 174-188

[10] S. Kirkpatrick, C. D. Gelatt, and M. P. Vecchi, "Optimization by simulated annealing," Science, 220 (4598), 1983, pp. 671-680

[11] M.B. M. De Koster, "Recent developments in warehousing", Working Paper, Rotterdam School of Management, Erasmus University, Rotterdam, 1998

[12] M. K. Lee, and E. A. Elsayed, "Optimization of warehouse storage capacity under a dedicated storage policy", International Journal of Production Research, 43 (9), 2005, pp. 1785-1805

[13] B. Molnar, and G. Lipovszki, "Multi-Objective Routing and Scheduling of Order Pickers in a Warehouse", International Journal of Simulation, 6(5), 2002, pp. 22-32

[14] J. Perl, and M. S. Daskin, "Warehouse location-routing problem", Transport. Res. 19(5), 1985, pp. 381-396

[15] V. V. Petinis, C. D. Tarantilis, and C. T. Kiranoudis, "Warehouse sizing and inventory scheduling for multiple stock-keeping products", International Journal of Systems Science, 36 (1), 2005, pp. 39-47

[16] J.I.U. Ruberico, and J. Ota, "Route generation for warehouse management using fast heuristics", Proceedings of IEEE/RSJ International Conference on Intelligent Robots and Systems, Sendai, Japan, 2004, pp. 2093-2098

[17] J.I.U. Rubrico, J. Ota , T. Higashi, and H. Tamura, "Scheduling multiple agents for picking products in a warehouse", Proceedings 2006 IEEE International Conference on Robotics and Automation, 2006 (ICRA 2006), 2006, pp. 1438- 1443

[18] D. R. Thompson, and G. L. Bilbro, "Sample-sort simulated annealing", Systems, Man and Cybernetics, Part B, IEEE Transactions, 35 (3), 2005, pp. $625-632$

[19] J.P. Van den Berg, and W.H.M. Zijm, "Models for warehouse management: classification and examples", International Journal of Production Research, 59, 1999, pp. 519-528. 